\title{
Shrinking microbubbles with microfluidics: mathematical modelling to control microbubble sizes
}

Received 00th January 20xx, Accepted 00th January 20xx

DOI: $10.1039 / \times 0 \times x 00000 x$

www.rsc.org/

\author{
A. Salari, ${ }^{\text {ade }}$ V. Gnyawali, ${ }^{\text {cde }}$ I.M. Griffiths, ${ }^{f}$ R. Karshafian, ${ }^{\text {bde M.C. Kolios, }}{ }^{\text {,de }}$ and S.S.H. Tsai ${ }^{\text {cde }} \dagger$
}

Microbubbles have applications in industry and life-sciences. In medicine, small encapsulated bubbles $(<10 \mu \mathrm{m})$ are desirable because of their utility in drug/oxygen delivery, sonoporation, and ultrasound diagnostics. While there are various techniques for generating microbubbles, microfluidic methods are distinguished due to their precise control and ease-offabrication. Nevertheless, sub-10 $\mu \mathrm{m}$ diameter bubble generation using microfluidics remains challenging, and typically requires expensive equipment and cumbersome setups. Recently, our group reported a microfluidic platform that shrinks microbubbles to sub-10 $\mu \mathrm{m}$ diameters. The microfluidic platform utilizes a simple microbubble-generating flow-focusing geometry, integrated with a vacuum shrinkage system, to achieve microbubble sizes that are desirable in medicine, and pave the way to eventual clinical uptake of microfluidically generated microbubbles. A theoretical framework is now needed to relate the size of the microbubbles produced and the system's input parameters. In this manuscript, we characterize microbubbles made with various lipid concentrations flowing in solutions that have different interfacial tensions, and monitor the changes in bubble size along the microfluidic channel under various vacuum pressures. We use the physics governing the shrinkage mechanism to develop a mathematical model that predicts the resulting bubble sizes and elucidates the dominant parameters controlling bubble sizes. The model shows a good agreement with the experimental data, predicting the resulting microbubble sizes under different experimental input conditions. We anticipate that the model will find utility in enabling users of the microfluidic platform to engineer bubbles of specific sizes.

\section{Introduction}

Microbubbles are currently used as ultrasound contrast agents, ${ }^{1}$ and also increasingly applied to drug delivery systems, ${ }^{2}$ as therapy agents, ${ }^{3}$ and as oxygen-transfer agents. ${ }^{4}$ In ultrasound imaging, microbubbles help provide important information about tissues, blood vessels, and drug pathways in the body. ${ }^{5,6}$ For example, in cancer diagnostics, tumor detection using traditional ultrasound is challenging because tumors and the surrounding tissues have similar acoustic impedance. Therefore, microbubbles are employed to increase the ultrasound contrast. The bubble gas-liquid interface has a high reflectance, which causes the bubbles to resonate under ultrasonic clinical frequency ranges of 1-10 MHz. Another example related to imaging is echo particle image velocimetry (echo-PIV), which is a non-invasive flow characterization method, where microbubbles act as flow tracers. ${ }^{1}$ This technique is based on backscattered ultrasound waves from bubbles, and provides accurate velocity measurements in cardiovascular flows. ${ }^{7,8}$

Apart from imaging, microbubbles are also used with ultrasound for therapeutic applications, including tissue/organ-specific drug and gene delivery. ${ }^{9-11}$ In these applications, the bubble surface (shell) may carry the molecule of interest. After bubbles are injected, ultrasonic waves at a specific frequency and amplitude impose pressure waves on the bubbles to release the drug molecules. ${ }^{12}$

In all the applications discussed previously, the size of the microbubbles is an important factor that can limit their

\footnotetext{
Biomedical Engineering Graduate Program, Ryerson University, Toronto, Canada.

${ }^{b}$ Department of Physics, Ryerson University, Toronto, Canada.

c Department of Mechanical and Industrial Engineering, Ryerson University, Toronto, Canada.

d Institute for Biomedical Engineering, Science and Technology (iBEST), Toronto, Canada.

e Keenan Research Centre, St. Michael's Hospital, Toronto, Canada.

$f$ Mathematical Institute, University of Oxford, Oxford, United Kingdom.

†E-mail:scott.tsai@ryerson.ca
}

functionality. ${ }^{13-15}$ However, commercially available microbubbles are often polydisperse, ${ }^{16,17}$ which leads to heterogeneous responses under ultrasound. For this reason, several microfluidics-based techniques have been developed to generate monodisperse microbubbles that have narrow size distributions. ${ }^{1,18-25}$ Techniques for microbubble generation in microfluidics use axisymmetric ${ }^{26}$ and symmetric ${ }^{1}$ flow-focusing channels, T-junctions, ${ }^{21}$ and microneedles. ${ }^{27}$ While these microfluidics-based methods create bubbles that are monodisperse, achieving sub $10-\mu \mathrm{m}$ diameter microbubbles, which are desirable in ultrasound imaging and therapy applications, ${ }^{28,29}$ is still challenging, and requires complex microfabrication techniques. ${ }^{19,30}$

To overcome these challenges, our group recently reported a microfluidic bubble shrinkage technique that generates sub-10 $\mu \mathrm{m}$ diameter microbubbles and achieves easily tunable microbubble sizes between 1-10 $\mu \mathrm{m}$. This technique is based on applying vacuum pressure in a polydimethylsiloxane (PDMS) based microfluidic chip to deplete air from the liquid-filled microchannels, so that suspended microbubbles shrink. ${ }^{25}$ The bubble-suspending microchannels are fabricated adjacent to vacuum microchannels, through which the negative pressure is applied.

In this manuscript, we develop a theoretical framework that relates the size of the microbubbles produced and the microfluidic system's experimental input parameters, such as the lipid concentration in the liquid, and the gas-liquid surface tension. We systematically characterize the microbubble shrinkage performance of this technique via a series of experiments. We then develop and experimentally validate a mathematical model to predict and control the microbubble shrinkage process. Finally, by asymptotic analysis, we reduce the full mathematical model, which is only solvable numerically, to a simplified form that we solve analytically to produce an explicit relationship between the microbubble size and the experimental input parameters. We anticipate that this simple mathematical expression will be useful for engineering specific microbubble sizes in many biomedical applications, such as contrast-enhanced ultrasound imaging. 


\section{Experimental Methods}

\section{Chemical Preparation}

Encapsulated microbubbles are generated in a microfluidic chip where air is used as the bubble core gas and a lipid solution as the encapsulation structure. We prepare two different lipid mixtures. In the first lipid mixture (M1), 1,2-distearoyl-sn-glycero-3phosphoethanolamine- $\mathrm{N}$-((polyethylene glycol-5000)folate) (ammonium salt) (DSPE-PEG5F-15) (Avanti Polar Lipids, Alabaster, AL, USA) is mixed with 1,2-distearoyl-sn-glycero-3-phosphocholine (DSPC) at 9:1 ratio in saline $\left(1.5 \mathrm{mg} \mathrm{mL}^{-1}\right)$. Glycerol (Sigma Aldrich Corporation, St. Louis, MO, USA) and pluronic F-68 (Fisher Scientific, Pittsburgh, PA, USA) are then added to the mixture in a 1:1:1 volumetric ratio to make the aqueous solution. The second lipid mixture (M2) is prepared similarly with the same molar/volumetric ratios, with the only difference that in the second mixture 1,2distearoyl-sn-glycero-3-phosphoethanolamine- $\mathrm{N}$ [Methoxy(Polyethylene glycol)-5000)folate) (ammonium salt) is used instead of DSPE-PEG5F-15. We measure the interfacial tension, $\sigma$, between the aqueous solution and air using the pendant-drop method. ${ }^{31}$

\section{Experimental Setup}

The details of experimental setup are reported in our previous manuscript. ${ }^{25}$ Briefly, we pattern a cross flow-focusing microfluidic design (Fig. 1) on a wafer substrate using a photolithography method. The height of all channels, including the vacuum channels, is $80 \mu \mathrm{m}$. We use the wafer as a mold for making PDMS microfluidic channels by soft lithography.

The injection of the aqueous solution is performed using a highprecision constant-flow-rate syringe pump (Harvard Instruments, Holliston, MA, USA). A pressure gauge (Omega Engineering Inc., Norwalk, Connecticut, USA) is used to regulate the air pressure. The vacuum pressure is adjusted using a Mityvac hand vacuum pump (Mityvac, St. Louis, MO, USA). We conduct the experiments by injecting the aqueous solution at a constant flow rate of $4 \mu \mathrm{L} \mathrm{min}^{-1}$ while air is supplied at a constant relative pressure of $27.6 \mathrm{kPa}$, and vacuum pressure is applied through the vacuum connections.

We image the bubbles at approximately the centre of each serpentine segment (red dashed rectangular area in Fig. 1) using a high-speed camera (Phantom M110, Vision Research, Wayne, NJ, USA) attached to an inverted microscope (Olympus Corp., Tokyo, Japan). The images are analysed by MATLAB (Mathworks, MA, USA) software using imfindcircles function to find the bubble size variation along the microfluidic channel.

The initial size of bubbles generated right after the flow-focusing junction is larger than the channel height, so bubbles are confined to discoid shapes. In order to accurately characterize bubble sizes, we use the bubbles' projected diameters to calculate the volume of the discoid bubbles, using a mathematical approach described elsewhere. ${ }^{25,32}$

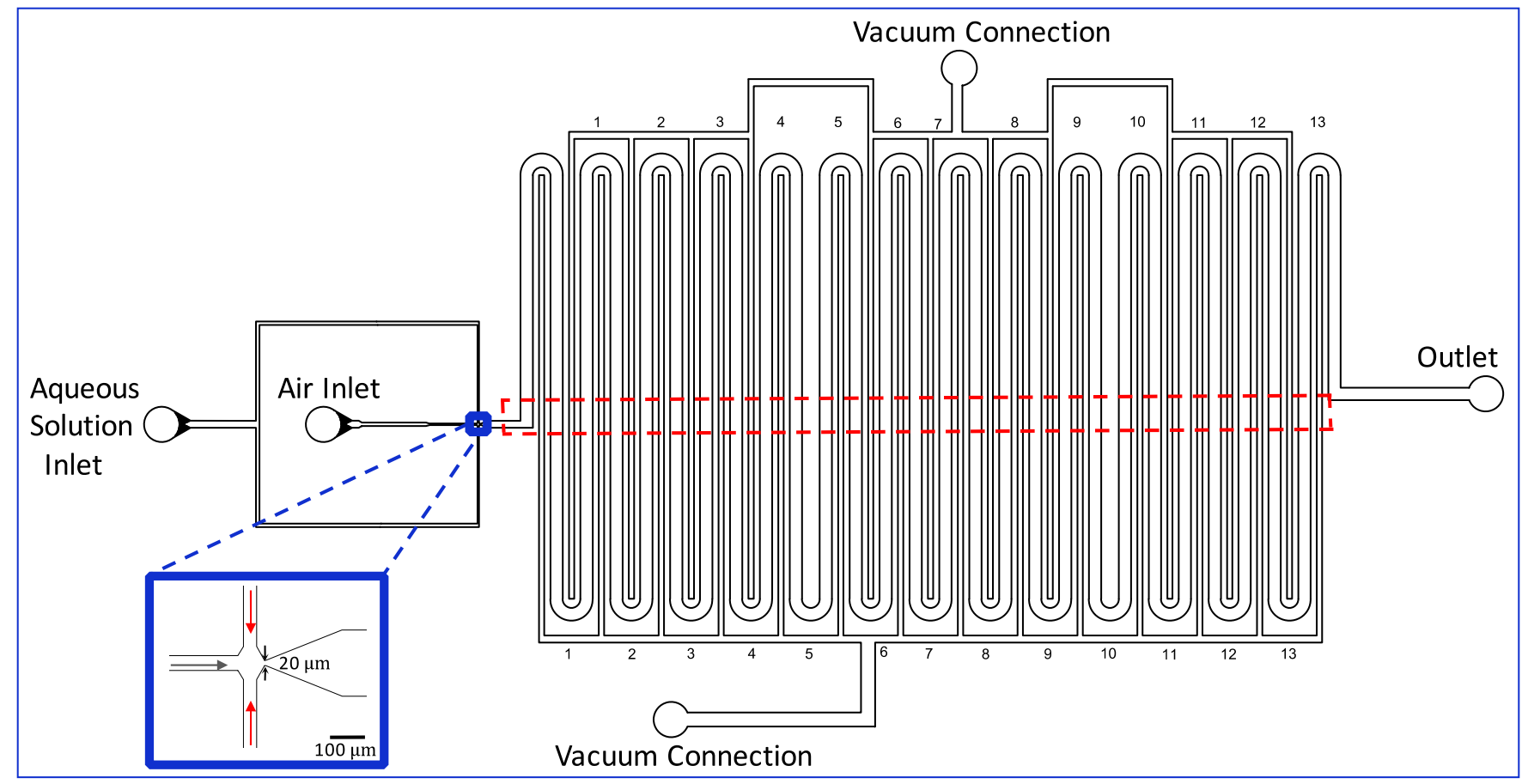

Fig. 1 A schematic design of the microfluidic system containing a serpentine liquid-filled channel with a total length of $350 \mathrm{~mm}$. Microbubbles are generated at a $20 \mu \mathrm{m}$ width junction, where flows of air (central channel, grey arrow) and aqueous solution (side channels, red arrows) meet orthogonally. Vacuum is applied through two connections to achieve a uniform vacuum pressure across the entire chip. The bubble size variation is monitored by moving the microscope within the area depicted by red dashed lines. Images are captured when bubbles pass through this area.

\section{Results and Discussions}

Experimental results
In order to study the effect of vacuum pressure on bubble shrinkage, we conduct experiments using a range of vacuum pressures $P_{v}=0$ to $-87 \mathrm{kPa}$, and with lipid mixture M1 (described in Experimental Methods). We also isolated the effect of other design parameters, such as gas core and channel dimensions, on 
bubble shrinkage by keeping them constant throughout our experiments. We note that the absolute pressure in the vacuum channel is equal to $P_{v}+P_{a t m}$, where $P_{a t m}$ is the atmospheric pressure.

The size of bubbles generated at microfluidic junctions is governed by the orifice geometry, gas pressure, liquid flow rate, liquid viscosity, and interfacial tension. 1,19,33 In this set of experiments, we maintain a constant initial microbubble radius $R=64 \pm 1 \mu \mathrm{m}$ (corresponding to a volume of $V=11 \times 10^{5} \mu \mathrm{m}^{3}$ ). This is the microbubble initial radius at the generation location, before exposure to vacuum shrinkage.

Fig. 2 shows the plot of microbubble volume $V$ versus position in the microchannel $l$. At the baseline vacuum pressure $P_{v}=0$, the bubbles experience a $\sim 60 \%$ reduction in volume by the end of the microchannel (after travelling approximately $350 \mathrm{~mm}$ ). Increasing the vacuum pressures cause further reduction in the bubble size. A maximum final size reduction of $\sim 99 \%$ is achieved for vacuum pressures $P_{v} \leq-60 \mathrm{kPa}$. We note that for vacuum pressure $P_{v} \leq$ $-60 \mathrm{kPa}$, the final bubble size is on the order of $\sim 1 \mu \mathrm{m}$, which approaches the diffraction limit of light, making the bubbles difficult to characterize.

We measure the aqueous solution air-liquid interfacial tension $\sigma=$ $36.60 \mathrm{mNm}^{-1}$. To systematically study the dependence of the final microbubble size on interfacial tension, we prepare aqueous solutions with two other interfacial tensions by diluting the original solution M1 in saline to achieve interfacial tensions $\sigma=$ 48.29 and $43.41 \mathrm{mNm}^{-1}$.

Fig. 3 shows the experimental results of bubble shrinkage using aqueous solutions with three different surface tensions. We find that the bubbles generated with solutions that have higher surface tensions are initially larger. When the interfacial tension $\sigma=$ $36.60 \mathrm{mNm}^{-1}$, bubbles are formed with an initial radius $R_{0}=$ $61 \mu \mathrm{m}$ (volume $V=9.46 \times 10^{5} \mu \mathrm{m}^{3}$ ), and their radius decreases by $\sim 62 \%$ by the end of the channel, when exposed to a vacuum pressure $P_{v}=-50 \mathrm{kPa}$. Under the same vacuum pressure, $P_{v}=$ $-50 \mathrm{kPa}$, microbubbles with initial radius $R_{0}=94 \mu \mathrm{m}$ (volume $V=$ $3.50 \times 10^{6} \mu \mathrm{m}^{3}$ ), and interfacial tension $\sigma=48.29 \mathrm{mNm}^{-1}$, shrink by $\sim 43 \%$.

When we apply a vacuum pressure $P_{v}=-87 \mathrm{kPa}$, bubbles generated using solutions with interfacial tensions $\sigma=$ $36.60 \mathrm{mNm}^{-1}$ and $43.41 \mathrm{mNm}^{-1}$ shrink to an approximate radius $R=0.5 \mu \mathrm{m}$ by the channel positions $l=250 \mathrm{~mm}$ and $325 \mathrm{~mm}$, respectively. No data is collected beyond that point. This shows $\sim 99 \%$ reduction in radius when interfacial tension $\sigma=$ $36.60 \mathrm{mNm}^{-1}$ and $\sim 68 \%$ reduction when interfacial tension $\sigma=$ $48.29 \mathrm{mNm}^{-1}$.

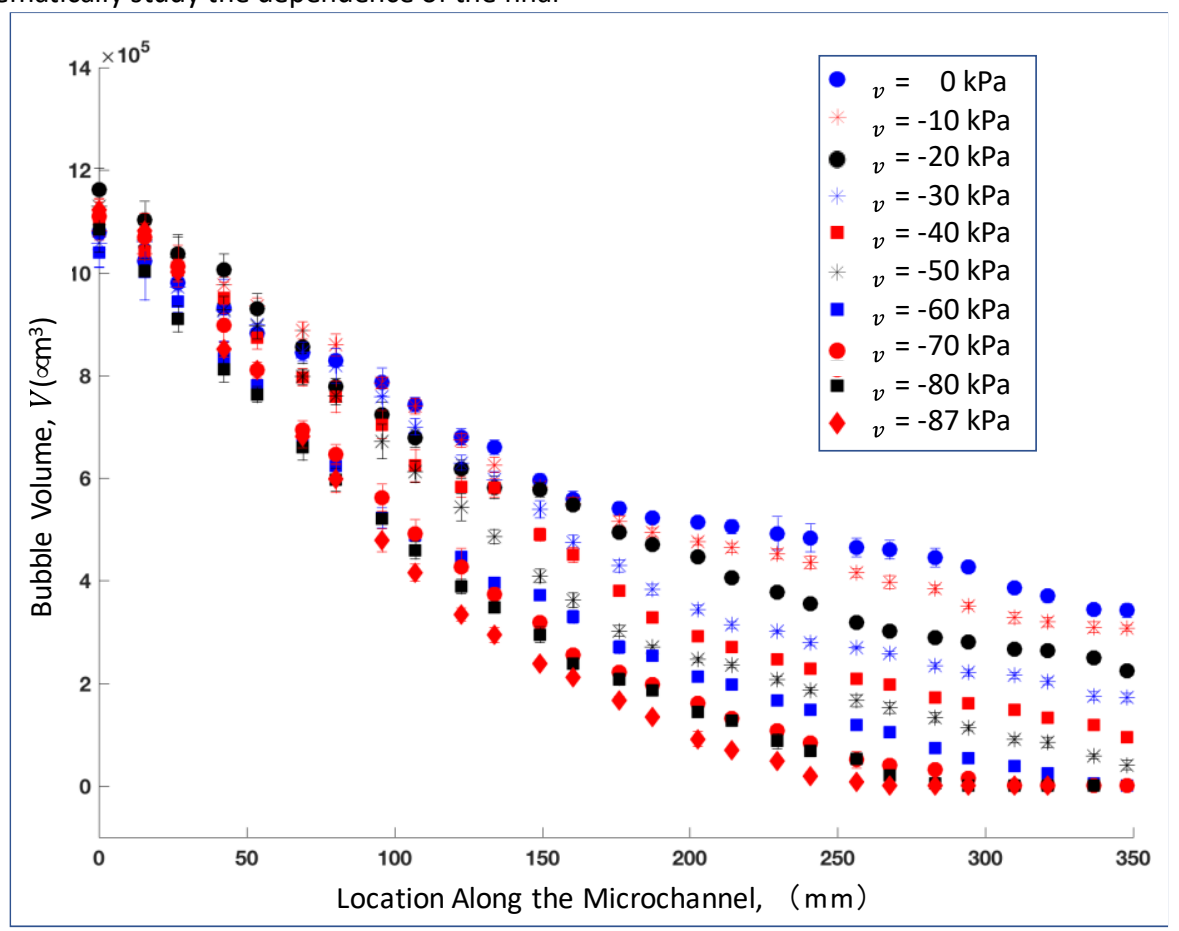

Fig. 2 A plot of the experimental results for the bubble volume $V$ versus the location $l$, along the microchannel at ten different vacuum pressures. The error bars indicate the standard deviations calculated by analysing $>10$ bubbles for each data point. In all experiments, the mixture surface tension, aqueous liquid flow rate, and air pressure are kept constant at $\sigma=36.60 \mathrm{mNm}^{-1}, 4 \mu \mathrm{L} \mathrm{min}{ }^{-1}$, and $27.6 \mathrm{kPa}$, respectively. The plot shows a monotonic trend of increasing microbubble shrinkage rate with increasing vacuum pressure. 

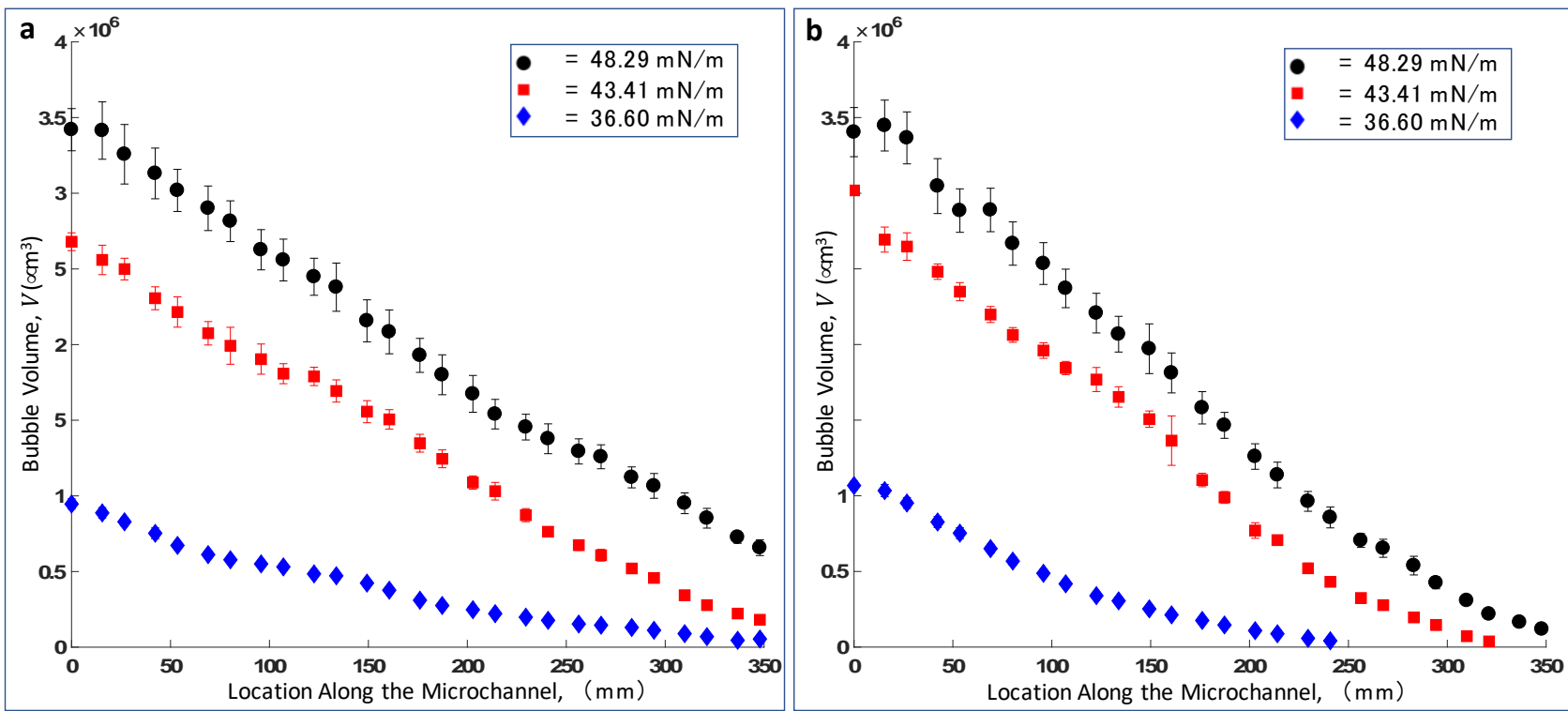

Fig. 3 Experimental results for the bubble volume $V$ versus the location along the microchannel $l$ for aqueous solutions with three different surface tensions. Error bars show standard deviations calculated by analysing $>10$ bubbles for each data point. Here, the vacuum pressure is held constant at (a) $P_{v}=-50 \mathrm{kPa}$, and (b) $P_{v}=-87 \mathrm{kPa}$. In all experiments, the aqueous liquid flow rate and air pressure are kept constant at $4 \mu \mathrm{Lmin}^{-1}$ and $27.6 \mathrm{kPa}$, respectively. Bubbles generated at higher surface tensions are initially larger and shrink more throughout the channel.

We also observe that changing the lipid mixture affects the shrinkage behaviour. Bubbles generated using mixture $\mathrm{M} 2$ demonstrate less shrinkage compared to bubbles formed from mixture M1. For example, the radius $R$ of bubbles generated using mixture $\mathrm{M} 2$ decreases by $\sim 52 \%$ by the end of the microchannel, while we observe a $\sim 99 \%$ reduction in radius $R$ for bubbles formed from mixture $\mathrm{M} 1$ using the same vacuum pressure $P_{v}=-87 \mathrm{kPa}$. Here, the surface tensions $\sigma$ of the two mixtures are similar, i.e. $\sigma=36.60 \mathrm{mNm}^{-1}$ for mixture $\mathrm{M} 1$ and $\sigma=37.66 \mathrm{mNm}^{-1}$ for mixture $\mathrm{M} 2$.

\section{Mathematical Modelling}

When microbubbles are generated in the aqueous solution, lipid molecules, which consist of a hydrophilic head and a hydrophobic tail, migrate towards and deposit onto the interface of the liquid/gas covering the bubbles, forming a self-assembled layer called a shell. ${ }^{18}$ The main function of shells in microbubble technology is to reduce the surface tension and extend the life-time of microbubbles from seconds to years. ${ }^{34}$ Microbubble stability is studied extensively in the literature because the lifetime and shell property of microbubbles are important in their application. ${ }^{18,35-42}$ Here, we apply some of the physical concepts from microbubble stability to model the shrinkage behaviour of the microbubbles in our microfluidic device, under vacuum pressure.

We apply a stability analysis of a typical microbubble with known shell encapsulation, and with a gas core of known transport properties. We neglect the effects of flow on the shrinkage once the bubble is generated, assuming that the bubble is continuously advected downstream with the flow. Therefore, the modelling is simplified to a microbubble stability analysis over time. This simplification assists in correlating the experimental data taken at each section of the serpentine channel to the time passed for the bubbles to reach to that section after they are generated at the flow-focusing junction.
Microbubble lifetimes were first mathematically modelled by Epstein and Plesset, where they considered a "clean" bubble, without encapsulation, in the bulk of a liquid and under diffusionlimited physics. ${ }^{43}$ Without encapsulation, a "clean" microbubble is unstable, dissolving in less than a second. Microbubbles stabilized by encapsulations can be modelled the same way, but with modified shell properties. ${ }^{36,42,44}$ Here, we follow the same approach to model our shrinking bubbles.

Assuming an encapsulated microbubble is fully immersed in a liquid, and the diffusion time-scale is much less than the dissolution time, the steady state conservation equation of core gas molecules in spherical coordinate is reduced to,

$$
\frac{1}{r^{2}} \frac{\mathrm{d}}{\mathrm{d} r}\left(r^{2} \frac{\mathrm{d} C}{\mathrm{~d} r}\right)=0
$$

where $C$, and $r$ are concentration (mole/volume) of the gas molecules in the aqueous bulk, and radial coordinate, respectively

As shown in Fig. 4, we can assume the following conditions for a single bubble with a radius $R$ and a shell thickness $\delta$, immersed in an aqueous solution,

$$
\begin{gathered}
C(r<(R-\delta))=C_{i n}, \\
C(r=(R-\delta))=C_{w}, \\
C(r=R)=C_{R}, \\
C(r \rightarrow \infty)=C_{f},
\end{gathered}
$$

where $C_{\text {in }}, C_{\text {wall }}, C_{R}$, and $C_{f}$ are the gas concentration inside the bubble, at the inner bubble wall (shell), at the bubble outer shell, and in the liquid bulk (aqueous solution) far from the bubble, respectively. Due to the laminar flow in the microfluidic channel, diffusion is the main transport mechanism responsible for gas release from bubbles to the vacuum channels, and thus the advection terms are neglected. Also, we neglect the effect of channel entrance and exit, and any bubble-bubble interactions on the gas transport mechanism. 
Conservation of mass through the shell provides one boundary condition for Eq. (1),

$$
-\left.k_{g} \frac{\mathrm{d} C}{\mathrm{~d} r}\right|_{r=R}=h_{g}\left(C_{w}-C_{R}\right)
$$

where $k_{g}$ and $h_{g}$ are the gas diffusion coefficient in the aqueous phase, and shell permeability coefficient, respectively.

Solving Eq. (1) with the boundary conditions $C(r \rightarrow \infty)=C_{f}$ and Eq. (3) gives,

$$
C(r)=R^{2}\left(\frac{C_{w}-C_{f}}{r\left(\frac{k_{g}}{h_{g}}+R\right)}\right)+C_{f} .
$$

Therefore,

$$
C_{R}=\frac{\frac{h_{g}}{k_{g}} R C_{w}+C_{f}}{1+R \frac{h_{g}}{k_{g}}} .
$$

Applying Ostwald's law and the ideal gas law, we obtain the following relation between the gas core pressure and inner shell concentration (Fig. 1c),

$$
\begin{aligned}
& C_{w}=L_{g} C_{i n} \\
& C_{\text {in }}=\frac{P_{\text {in }}}{R_{G} T},
\end{aligned}
$$

where $L_{g}, P_{i n}, R_{G}$, and $T$ are the Ostwald coefficient of the core gas, gas pressure inside the bubble, universal gas constant, and gas temperature, respectively.

Similarly, as shown in Fig. $4 \mathrm{~b}$, the concentration of gas in the aqueous solution far from the bubble, $C_{f}$, is related to the concentration at the fluid channel wall outside of the aqueous phase, $C_{c h}$,

$$
C_{f}=f L_{g} C_{c h},
$$

where, $f$ is typically defined as the level of saturation of the aqueous solution. However, since the level of saturation is not homogenous throughout the liquid in our system, we refer to $f$ as an effective level of saturation. We hypothesize that, at each cross section of the channel, the nominal level of saturation will be approximately unity at the bubble-liquid interface, greater than unity at the channel wall, and below unity throughout the rest of the liquid.

The nominal level of saturation also varies along the aqueous solution channel. For example, the value near the inlet may be different from that close to outlet. This inhomogeneity may arise from the effect of the pressure drop inside the aqueous solution, ${ }^{45,46}$ and diffusion through the PDMS bulk. ${ }^{6}$ Therefore, for simplicity and convenience, we define $f$ as an effective level of saturation, which we use as a fitting parameter in our model, to account for all of the inhomogeneity in liquid saturation mentioned above.
It has been shown in the literature that for high gas or air permeability (diffusivity, porosity, and solubility) ${ }^{47}$ and thin PDMS membranes ( $175 \mu \mathrm{m}$ wall thickness in our case), equilibrium across the thicknesses can be achieved very quickly, often within a few seconds. ${ }^{48}$ Neglecting the concentration difference across the bulk PDMS, i.e. assuming $C_{c h} \approx C_{a}$, and assuming the gas inside the vacuum channel is an ideal gas, we can write,

$$
C_{f}=f L_{g} \frac{P_{a}}{R_{G} T^{\prime}}
$$

where $P_{a}$ is the pressure inside the vacuum channel and is determined by the vacuum pressure, $P_{v}$,

$$
P_{a}=P_{a t m}+P_{v}
$$

To relate the bubble size to the gas concentration, we apply the mass conservation equation for the gas core assuming a bubble volume of $V=4 / 3 \pi R^{3}$ and surface area of $A=4 \pi R^{2}$,

$$
\frac{\mathrm{d}}{\mathrm{d} t}\left(\frac{4}{3} \pi R^{3} C_{\text {in }}\right)=-\left(4 \pi R^{2}\right) h_{g}\left(C_{w}-C_{R}\right)
$$

Due to interfacial tension effects on the bubble shell, Laplace's equation governs the pressure difference across the shell. We note that, for an encapsulated microbubble, the surface tension, $\sigma$, is a function of bubble radius, $R$, and therefore, a dilatational surface elasticity $E_{s}$ can be defined as the derivative of surface tension with respect to fractional change in the interfacial area, ${ }^{36,41,42,49}$

$$
\begin{gathered}
P_{\text {in }}-P_{f}=\frac{2 \sigma(R)}{R}, \\
\sigma(R)=\sigma_{0}+E_{S}\left(\left(\frac{R}{R_{0}}\right)^{2}-1\right),
\end{gathered}
$$

where $P_{i n}, R_{0}$, and $\sigma_{0}$ are the pressure inside bubble, initial radius of the stress-free bubble conformation, and initial stress-free interfacial tension of the bubble, respectively.

Now, we can substitute Eq. (11) into Eq. (6), assuming $P_{f}$ is equal to the ambient pressure, $P_{\text {atm }}$,

$$
\begin{gathered}
C_{w}=L_{g} \frac{P_{a t m}+\frac{2 \sigma_{0}}{R}+\frac{2 E_{S}}{R}\left(\left(\frac{R}{R_{0}}\right)^{2}-1\right)}{R_{G} T}, \\
C_{\text {in }}=\frac{P_{a t m}+\frac{2 \sigma_{0}}{R}+\frac{2 E_{s}}{R}\left(\left(\frac{R}{R_{0}}\right)^{2}-1\right)}{R_{G} T} .
\end{gathered}
$$

Similarly, we can substitute Eqs. (8) and (12) into Eq. (5),

$$
\begin{aligned}
C_{R}=\frac{L_{g}}{R_{G} T\left(1+R \frac{h_{g}}{k_{g}}\right)}\left(\frac { h _ { g } } { k _ { g } } R \left(P_{a t m}+\frac{2 \sigma_{0}}{R}\right.\right. \\
\left.\left.+\frac{2 E_{s}}{R}\left(\left(\frac{R}{R_{0}}\right)^{2}-1\right)\right)+f P_{a}\right) .
\end{aligned}
$$

Finally, substituting Eqs. (12) and (13) into (10), we obtain the governing equation for the time-dependent bubble radius as follows,

$$
\frac{\mathrm{d} R}{\mathrm{~d} t}=-\frac{3 k_{g} L_{g}}{\frac{k_{g}}{h_{g}}+R}\left(\frac{(1-f) P_{a t m}-f P_{v}+\frac{2 \sigma_{0}}{R}+\frac{2 E_{s} R}{R_{0}{ }^{2}}-\frac{2 E_{s}}{R}}{3 P_{a t m}+\frac{4 \sigma_{0}}{R}+\frac{8 E_{s} R}{R_{0}{ }^{2}}-\frac{4 E_{s}}{R}}\right) .
$$


Eq. (14) can be nondimensionalized by defining the following dimensionless parameters,

$$
\begin{array}{cc}
\hat{t}=\frac{3 t k_{g} L_{g}}{R_{0}{ }^{2}}, & \hat{R}=\frac{R}{R_{0}}, \\
\widehat{k_{g}}=\frac{k_{g}}{h_{g} R_{0}}, & \hat{\sigma}=\frac{2 \sigma_{0}}{P_{a t m} R_{0}}, \\
\hat{E}=\frac{2 E_{s}}{P_{a t m} R_{0}}, & \widehat{P_{v}}=\frac{P_{v}}{P_{a t m}},
\end{array}
$$

where $\hat{t}, \hat{R}$, and $\widehat{P}_{v}$ are dimensionless values representing timescale, bubble radius, and vacuum pressure, respectively. $\widehat{k_{g}}, \hat{\sigma}$, and $\hat{E}$ are dimensionless values representing diffusion coefficient, interfacial tension, and elasticity, respectively.

This allows us to write Eq. (14) as,

$$
\frac{\mathrm{d} \hat{R}}{\mathrm{~d} \hat{t}}=-\frac{1}{\widehat{k_{g}}+\hat{R}}\left(\frac{(1-f)-f \widehat{P_{v}}+\frac{\hat{\sigma}}{\hat{R}}+\hat{E} \hat{R}-\frac{\hat{E}}{\hat{R}}}{3+\frac{2 \hat{\sigma}}{\hat{R}}+4 \hat{E} \hat{R}-\frac{2 \hat{E}}{\hat{R}}}\right) .
$$

In addition to modelling bubble size changes over time, we also use COMSOL Multiphysics software to conduct a 2D numerical simulation of typical gas (air) convection through a long $(350 \mathrm{~mm}$ ) microfluidic channel filled with fully saturated liquid (water) at ambient pressure. Here, we apply three different concentration conditions imposed by Ostwald's law on the PDMS wall for each

vacuum pressure (i.e. $P_{v}=0,-50,-87 \mathrm{kPa}$ ). Fig. 5 shows the numerical solution of concentration variation along the centreline of a $350 \mathrm{~mm}$ long liquid filled microfluidic channel and the 2D geometry used in the simulation. The simulations show that, regardless of the vacuum pressure magnitude, the PDMS wall thickness is small enough that the air concentration in the aqueous flow attains its equilibrium value in less than $\sim 15 \%$ of the channel length. This effect is thus ignored in our modelling, and can be made smaller if the PDMS wall between the liquid filled microfluidic channel and the vacuum channel is thinner.

We can solve Eq. (16) by separation of variables, subject to the initial condition $\hat{R}=1$ to give,

$$
=\int_{1}^{\hat{t}(\hat{R})}-\left(\widehat{k_{g}}+\tilde{R}\right)\left(\frac{3+\frac{2 \hat{\sigma}}{\tilde{R}}+4 \hat{E} \tilde{R}-\frac{2 \hat{E}}{\tilde{R}}}{(1-f)-f \widehat{P}_{v}+\frac{\hat{\sigma}}{\tilde{R}}+\hat{E} \tilde{R}-\frac{\hat{E}}{\tilde{R}}}\right) d \tilde{R} .
$$

The integral on the right-hand side of Eq. (17) can be evaluated analytically to give,

$$
\hat{t}(\hat{R})=g(\hat{R})-g(1)
$$

where

$$
\begin{aligned}
& g(\hat{R})=\left[4 \widehat{E}^{3} \widehat{k_{g}}+\left(-1+f+f \widehat{P}_{v}\right)^{2}\left(-1+4 f\left(1+\widehat{P}_{v}\right)\right)+\widehat{E}\left(\widehat{k_{g}}\left(1-5 f\left(1+\widehat{P_{v}}\right)+4 f^{2}\left(1+\widehat{P_{v}}\right)^{2}\right)-\right.\right. \\
& \left.\left.2\left(-2+5 f\left(1+\widehat{P}_{v}\right)\right) \hat{\sigma}\right)+2 \hat{E}^{2}\left(5 f\left(1+\widehat{P}_{v}\right)-2\left(1+\widehat{k_{g}} \hat{\sigma}\right)\right)\right] \times \\
& \operatorname{Arctan}\left(\frac{1-f\left(1+\widehat{P}_{v}\right)+2 \hat{E} \hat{R}}{\sqrt{-4 \hat{E}^{2}-\left(-1+f+f \widehat{P}_{v}\right)^{2}+4 \hat{E} \hat{\sigma}}}\right) \\
& {\left[\hat{E}^{2} \sqrt{-4 \hat{E}^{2}-\left(-1+f+f \widehat{P}_{v}\right)^{2}+4 \hat{E} \hat{\sigma}}\right]} \\
& \frac{\left[\left(1+2 \widehat{E}^{2}-5 f\left(1+\widehat{P}_{v}\right)+4 f^{2}\left(1+\widehat{P_{v}}\right)^{2}+\hat{E}\left(\widehat{k_{g}}\left(-1+4 f\left(1+\widehat{P_{v}}\right)\right)-2 \hat{\sigma}\right)\right) \log \left(-\left(-1+f+f \widehat{P_{v}}\right) \hat{R}+\hat{E}\left(-1+\hat{R}^{2}\right)+\hat{\sigma}\right)\right]}{\left[2 \hat{E}^{2}\right]} \text {. }
\end{aligned}
$$

Equation (18) gives the dimensionless time at which a desired encapsulated microbubble radius is attained for a given vacuum pressure, an aqueous solution of known surface tension, gas diffusion coefficient, and effective level of gas saturation, and a bubble shell of known permeability and elasticity. We take vacuum pressure $P_{v}=0$ as the control experiment to obtain values for our fitting parameters, which are shell properties $\left(h_{g}, E_{s}\right)$ and the effective level of saturation $f$. We find a good agreement with the experimental data in predicting the bubble size versus time when $f=0.62, h_{g}=2.6 \times 10^{-5} \mathrm{~ms}^{-1}$, and $E_{s}=9 \times 10^{-3} \mathrm{Nm}^{-1}$. These values are all within the range of commonly used values in literature..$^{36,42,44}$ We note that we maintain the same fitting parameter values for all experiments, including for solutions with different surface tensions and experiments under various vacuum pressures (Fig. 6). We also keep the following parameters constant: $L_{g}=1.7 \times 10^{-2}$ and $k_{g}=2.05 \times 10^{-9} \mathrm{~m}^{2} \mathrm{~s}^{-1} .{ }^{36}$
The phenomenon of bubble shrinkage in the absence of vacuum pressure is reported in earlier literature when the saturation $f<$ $1,36,50$ and observed here in our experiments. Our hypothesis for this somewhat surprising observation is the following.

One part of our fabrication process leaves the PDMS bulk inside a vacuum chamber for a few minutes to perform plasma-treatment on the channel surfaces. In order to maintain constant surface chemistry in all of the microfluidic devices we use, all of our experiments are conducted within $30 \mathrm{~min}$ of this treatment step. Therefore, the PDMS bulk is always degassed at the time of each experiment, causing the PDMS bulk to absorb air from all directions as well as from the aqueous solution at a very low rate, yielding the effective level of saturation $f<1$.

To test this hypothesis, we perform a similar experiment with an air-saturated PDMS device at vacuum pressure $P_{v}=0$. As 
illustrated in Supplementary Information Fig. S1, the shrinkage of the bubbles in the air-saturated PDMS device, when vacuum pressure $P_{v}=0$, is almost negligible, suggesting that the usage of degassed PDMS device in our experiments is the main contributor to our observed bubble shrinkage at vacuum pressure $P_{v}=0$.

When $P_{v}>0$, the highest flux of gas mass transfer occurs in the direction from the aqueous solution to the vacuum channel, which has the lowest resistance to mass transfer, causing the bubble to shrink at a higher rate.
In general, depending on the shell properties and effective level of saturation, the bubbles will either reach a stable final radius, or dissolve entirely. Our results show that, under the above-

mentioned conditions, bubble shrinkage continues if the vacuum pressure is applied. One can adjust the channel length in a way that, once the bubbles reach to the desired final size, they can be collected. Alternatively, the vacuum pressure can be adjusted accordingly so that the bubbles reach the channel outlet at the desired size.

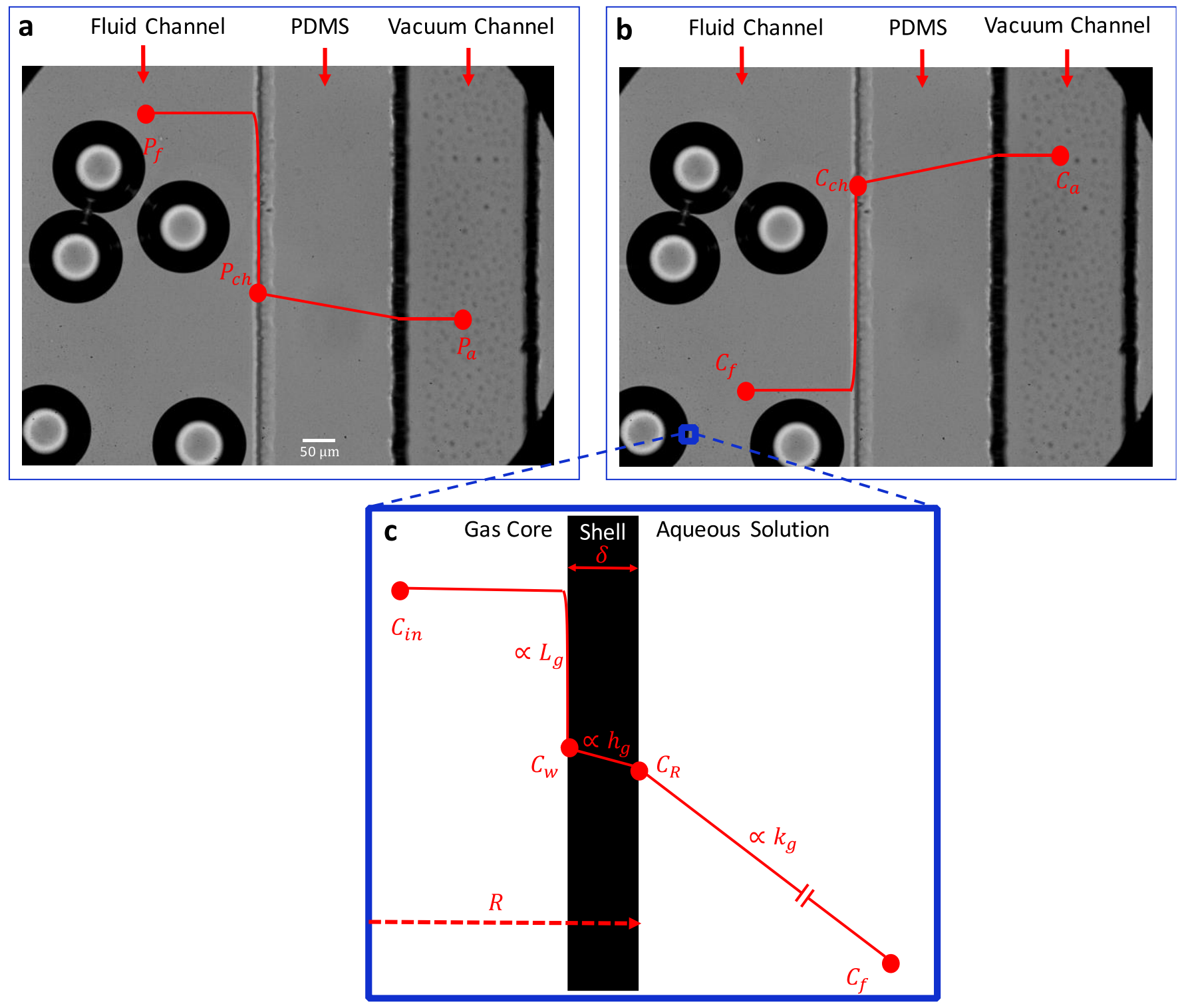

Fig. 4 Microscopy images showing the fluid channel, PDMS bulk, and the vacuum channel. (a) The schematic profile overlaid on the images, of absolute pressure distribution and (b) the concentration distribution across the microfluidic channel. The changes of absolute pressure and air concentration through the PDMS bulk is negligible. Ostwald's law governs the relationship between the absolute pressure and air concentration in the fluid channel, and pressure and air concentration in the bulk PDMS. (c) A schematic diagram of the concentration changes across a microbubble shell. 


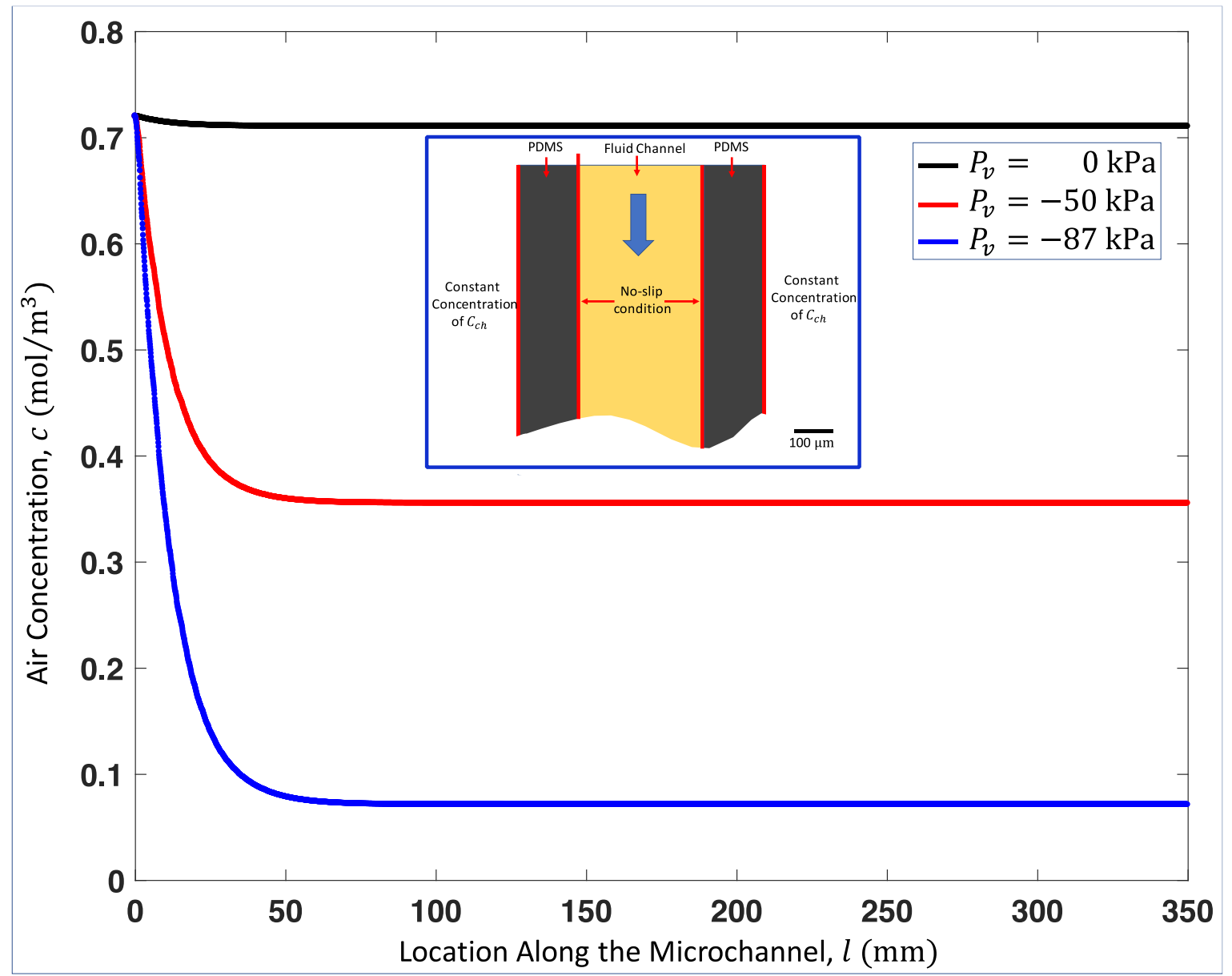

Fig. 5 Simulation results representing the concentration of dissolved air along the channel centreline at three different vacuum pressures, $P_{v}=0,-50$, and $-87 \mathrm{kPa}$. In simulations, we assume that the microfluidic channel is filled with water only, and thus the effect of bubble flow is neglected. The effect of channel entrance on the air concentration within the liquid is diminished once the liquid travels $50 \mathrm{~mm}$ along the channel length. The inset shows the 2D geometry used in the simulation and the boundary conditions applied. 

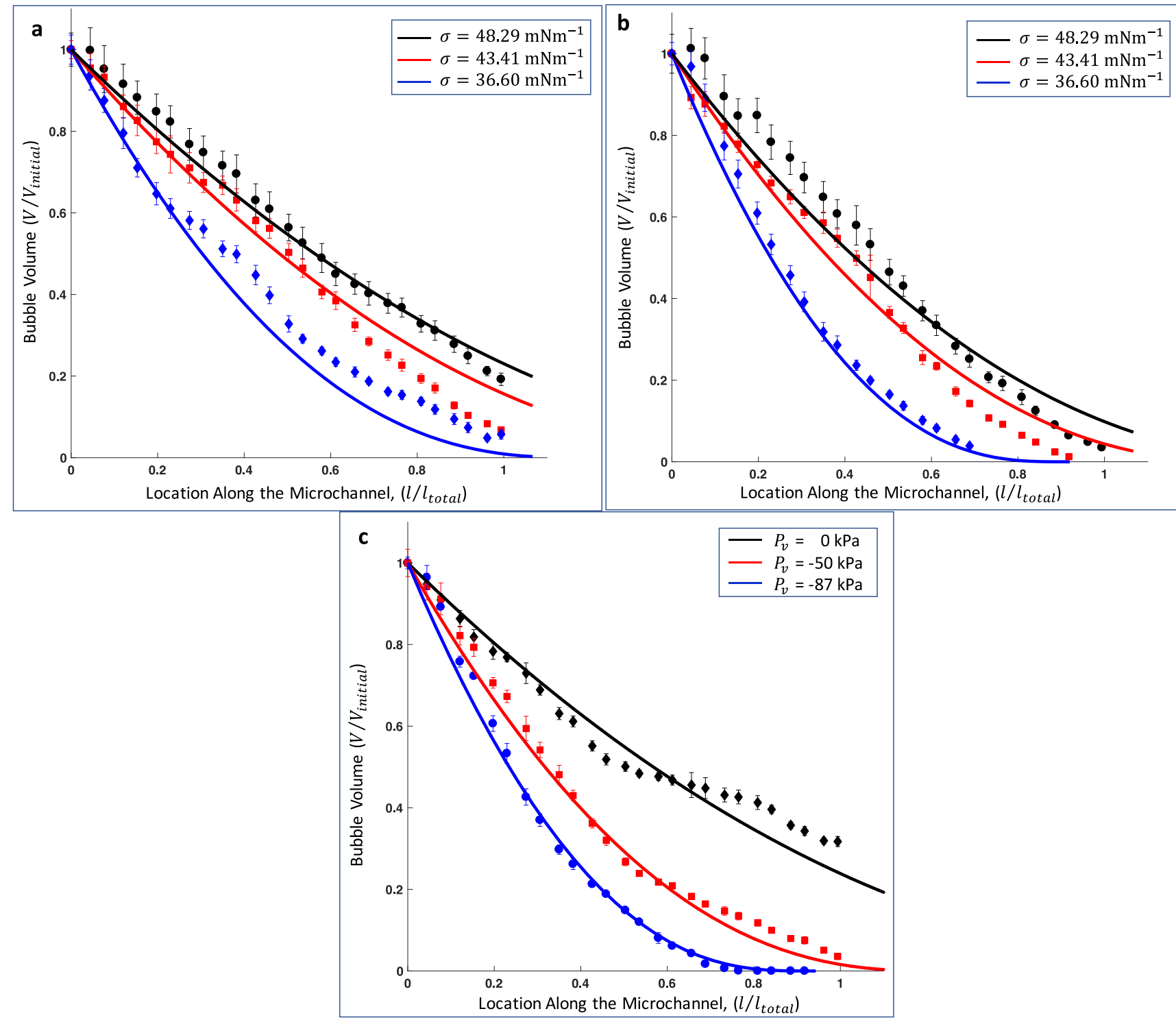

Fig. 6 Dimensionless experimental and modelling results for the bubble volume $V / V_{\text {initial }}$ versus the location along the channel $l / l_{\text {total }}$. The results are shown for different surface tensions $\sigma$, while vacuum pressures (a) $P_{v}=-50 \mathrm{kPa}$, and (b) $P_{v}=-87 \mathrm{kPa}$. (c) Three different vacuum pressures with constant surface tension $\sigma=36.60 \mathrm{~m} \mathrm{Nm}^{-1}$. Data points state experimental results, while the solid lines represent the numerical solution of the mathematical model. The modelling results demonstrate a very good agreement with the experimental data. Here, initial bubble volume $V_{\text {initial }}=4 / 3 \pi R_{0}{ }^{3}$ and the microchannel length $l_{\text {total }}=350 \mathrm{~mm}$. In (a) and (b), initial bubble radius $R_{0}=89 \mu \mathrm{m}, 83 \mu \mathrm{m}$, and $61 \mu \mathrm{m}$, correspond to surface tensions $\sigma=48.29 \mathrm{mNm}^{-1}, 43.41 \mathrm{mNm}^{-1}$, and $36.60 \mathrm{mNm}^{-1}$, respectively. In (c), the initial bubble radius $R_{0}=63 \mu \mathrm{m}$.

In all the experiments, the dimensionless surface tension $\hat{\sigma}$ and elasticity $\hat{E}$ are small quantities. Typically, $\hat{\sigma} \simeq O\left(10^{-2}\right)$ and $\hat{E} \simeq$ $\left.O\left(10^{-3}\right)\right)$. In this limit, Eq. (18) is reducible to the simple expression,

$$
\hat{R}(\hat{t})=-\widehat{k_{g}}+\sqrt{\left(1+\widehat{k_{g}}\right)^{2}+\frac{2}{3}\left(\left(1+\widehat{P_{v}}\right) f-1\right) \hat{t}}
$$

which is in excellent agreement with the full analytical solution (Fig. 7). 


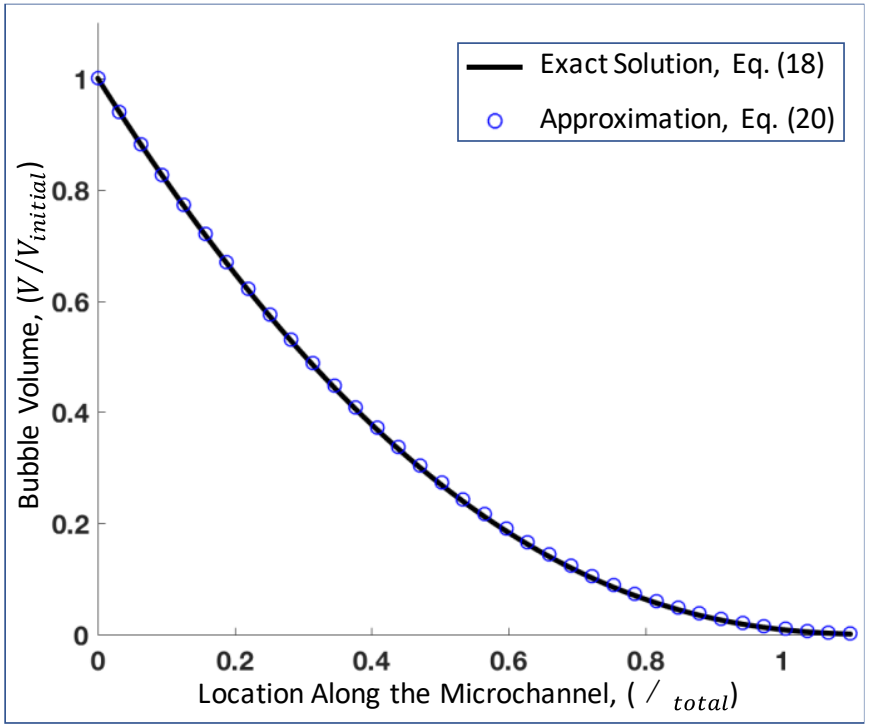

Fig. $7 \mathrm{~A}$ plot of the dimensionless bubble volume $V / V_{\text {initial }}$ versus the position along the channel $l / l_{\text {total }}$. Here, the dimensionless parameters are $\hat{\sigma}=1.18 \times 10^{-2}, \vec{P}_{v}=-0.5, \hat{E}=2.90 \times 10^{-3}$, $\widehat{k_{g}}=1.27$, and $f=0.62$. The approximated solution (Eq. 20) is almost identical to the exact solution (Eq. 18).

Thus, this simplified expression of the bubble radius (Eq. (20)) is valid for all liquid solutions that have relatively small surface tension $\hat{\sigma}$ and dilatational surface elasticity $\hat{E}$. In these circumstances, our analysis shows that for a given initial bubble radius, the effect of liquid surface tension and dilatational surface elasticity of the bubble on the shrinkage rate is negligible.

The agreement indicates that our shrinkage model is versatile enough to be applied to microfluidic systems where bubbly flows are mixed with flows with different surface tensions, such as biofluids. However, as shown in Figs. $6 a$ and $6 \mathrm{~b}$, we still observe different shrinkage rates upon changing the liquid surface tension $\sigma$ since changes in the surface tension manifest themselves through changes in the initial bubble radius $R_{0}$.

Eqs (18), (19) and (20) together provide a clear and powerful method for determining the operating conditions required to generate any desired bubble size. Eqs. (18) and (19) explicitly encapsulate all of the physical variables within the system, and the way in which they influence the bubble evolution. As a result, even if experimental limitations provide bounds on what can be physically achieved for one particular parameter, this equation indicates how the other physical parameters can be manipulated to produce a desired bubble size.

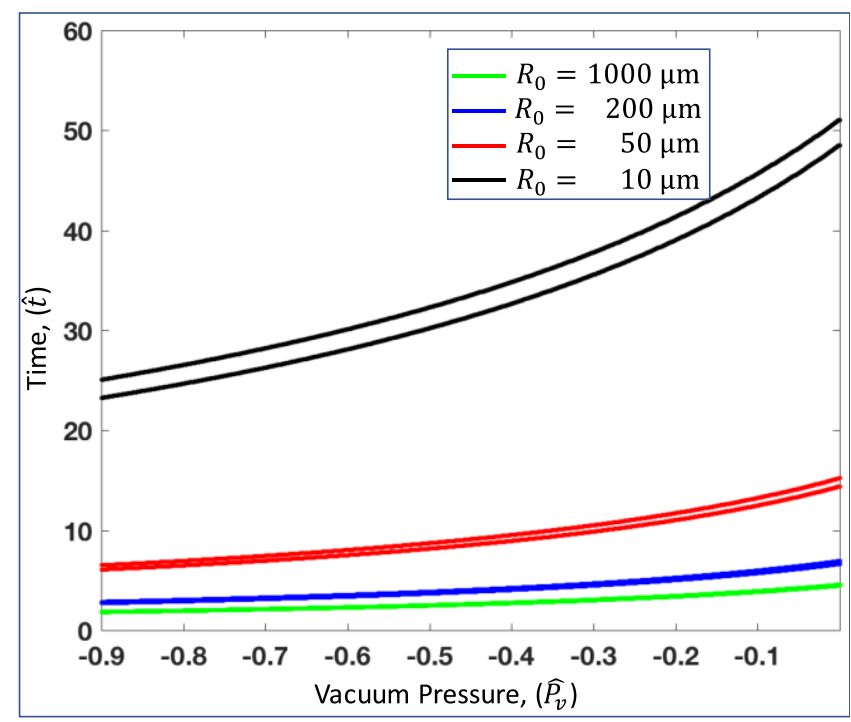

Fig. 8 The dependence of shrinkage time required for bubbles to reach a radius $\hat{R}=0.01$ (upper curve) and $\hat{R}=0.1$ (lower curve) given an initial radius, $R_{0}$, on vacuum pressure. The vacuum pressure $\widehat{P}_{v}$ is more effective on initially smaller bubbles.

The further reduced form of Eq. (20) takes the analytical expression a step further by providing an explicit form for the bubble radius as a function of the key parameters in this particular microfluidic system, namely the diffusion coefficient, effective level of saturation, shell permittivity, Ostwald's coefficient, and vacuum pressure. The result provides a mechanism for rapid prediction of the appropriate operating regimes required to fabricate bubbles with desired radii.

As one such illustration of the simplicity of Eq. (20), we can determine the bubble size reduction over a specific time interval. Fig. 8 shows the dimensionless shrinkage time required for bubble shrinkage to $\hat{R}=0.01$ and $\hat{R}=0.1$ versus vacuum pressure for bubbles with different initial sizes. This information is useful from an engineering standpoint. For instance, this model informs us that we require a dimensionless time of $\hat{t}=30$ to shrink a bubble with initial size of $R_{0}=10 \mu \mathrm{m}$ to a final size of $R=0.1 \mu \mathrm{m}$ if a vacuum of $\widehat{P}_{v}=-0.6$ is applied, while we can achieve a final size of $R=$ $1 \mu \mathrm{m}$ if a vacuum of $\widehat{P_{v}}=-0.5$ is applied. The amount of time required can be converted to the channel length and be used for designing a microfluidic shrinkage setup.

\section{Conclusion}

In this paper, we characterize a microfluidic microbubble shrinkage technique that utilizes the gas permeability of PDMS microchannels and the permeability of bubble encapsulations, to shrink microbubbles. We develop a mathematical model that predicts the size of the resulting microbubbles, and find a very good agreement between the model and experimental data, under different experimental conditions. We expect that this model, especially in the simplified closed-form version, will find utility in the engineering and manufacturing of microbubbles of specific sizes.

Although our microfluidic bubble-shrinking device results in low volumes of gas surrounded by relatively large volume of liquid (i.e. low number of bubbles per volume of liquid), multiple techniques can be implemented to increase the throughput. These techniques include on-chip multiplexing of the bubble-generation orifices, ${ }^{51}$ 
reducing the width $(<5 \mu \mathrm{m})$ of the flow-focusing orifice using precise microfabrication techniques, ${ }^{39}$ and using an on-chip bubble separator to remove the carrier liquid and control the number of bubbles per liquid volume. ${ }^{52,53}$ Our modeling and experimental results reported in this paper can also be used to better understand the physical mechanism behind vacuum-based bubble shrinkage, and enable the design of a device capable of reducing the bubble sizes down to order $1 \mu \mathrm{m}$ or less, thus potentially offering a submicron bubble generation technique. In such a device, one can predict the amount of vacuum pressure required for a specific type of lipid solution in order that a desired bubble size is collected at the outlet. Channel design optimization, investigation of other types of bubble encapsulations, and integration with submicron bubble characterization techniques offer future research directions to pave the way towards making inexpensive and easy-to-fabricate stabilized nanobubbles.

\section{Acknowledgement}

AS, VG, SSHT, MCK, and RK acknowledge affiliation with the Institute for Biomedical Engineering, Science, and Technology (iBEST)-a partnership between Ryerson University and St. Michael's Hospital. IMG gratefully acknowledges support from the Royal Society through a University Research Fellowship. SSHT acknowledges funding support from the Discovery grants program of the Natural Sciences and Engineering Research Council of Canada (NSERC). MCK and RK thank NSERC and the Canadian Institutes of Health Research (CIHR) for funding from the Collaborative Health Research Projects (CHRP) program.

\section{References}

A. Salari, M. B. Shafii and S. Shirani, J Fluid Eng T ASME, 2013, 135, 34501

J. S. Oh, Y. S. Kwon, K. H. Lee, W. Jeong, S. K. Chung and K. Rhee, Comput. Biol. Med., 2014, 44, 37-43.

Y. Liu, F. Yang, C. Yuan, M. Li, T. Wang, B. Chen, J. Jin, P. Zhao, J. Tong, S. Luo and N. Gu, ACS Nano, 2017, 11, 15091519.

J. R. Eisenbrey, L. Albala, M. R. Kramer, N. Daroshefski, D. Brown, J. Bin Liu, M. Stanczak, P. O'Kane, F. Forsberg and M. A. Wheatley, Int. J. Pharm., 2015, 478, 361-367.

S. Dindyal and C. Kyriakides, Recent Pat. Cardiovasc. Drug Discov., 2011, 6, 27-41.

J. R. Lindner, Nat. Rev. Drug Discov., 2004, 3, 527-533.

F. Zhang, C. Lanning, L. Mazzaro, A. J. Barker, P. E. Gates, W. D. Strain, J. Fulford, O. E. Gosling, A. C. Shore, N. G. Bellenger, B. Rech, J. Chen, J. Chen and R. Shandas, Ultrasound Med. Biol., 2011, 37, 450-464.

8 M. Siciliano, F. Migliore, L. Badano, E. Bertaglia, G. Pedrizzetti, S. Cavedon, A. Zorzi, D. Corrado, S. Iliceto and D. Muraru, Europace, 2016, 350, 2140-50.

Y. Lv, L. Hao, W. Hu, Y. Ran, Y. Bai and L. Zhang, Sci. Rep.,

2016, 6, 29321.

X. Zhu, J. Guo, C. He, H. Geng, G. Yu, J. Li, H. Zheng, X. Ji and F. Yan, Sci. Rep., 2016, 6, 21683.

A. Burgess and K. Hynynen, in Advances in experimental medicine and biology, 2016, vol. 880, pp. 293-308.

D. Carugo, M. Aron, E. Sezgin, J. Bernardino de la Serna, M. K. Kuimova, C. Eggeling and E. Stride, Biomaterials, 2017, 113, 105-117.

S. Sirsi and M. Borden, Bubble Sci Eng Technol., 2010, 1, 317.

S. R. Sirsi and M. A. Borden, Theranostics, 2012, 2, 12081222.

S. R. Sirsi and M. A. Borden, Adv. Drug Deliv. Rev., 2014, 72, 3-14.

S. Wang, G. Samiotaki, O. Olumolade, J. A. Feshitan, E. E. Konofagou, E. E. Konofagou, T. van der Steen, M. Versluis and N. de Jong, Ultrasound Med. Biol., 2014, 40, 130-7.

S. Sirsi, J. Feshitan, J. Kwan, S. Homma and M. Borden, Ultrasound Med. Biol., 2010, 36, 935-948.

M. Lee, E. Y. Lee, D. Lee and B. J. Park, Soft Matter, 2015, 11, 2067-2079.

K. Hettiarachchi, E. Talu, M. L. Longo, P. A. Dayton and A. P. Lee, Lab Chip, 2007, 7, 463-8.

J. Wan, A. Bick, M. Sullivan and H. A. Stone, Adv. Mater., 2008, 20, 3314-3318.

M. Parhizkar, E. Stride and M. Edirisinghe, Lab Chip, 2014, 14, 2437.

J. H. Xu, S. W. Li, G. G. Chen and G. S. Luo, AIChE J., 2006, 52, 2254-2259.

J. Fiabane, P. Prentice and K. Pancholi, Biomed Res. Int., 2016, 2016.

Z. Z. Chong, S. B. Tor, N. H. Loh, T. N. Wong, A. M. GañánCalvo, S. H. Tan and N.-T. Nguyen, Lab Chip, 2015, 15, 996999.

V. Gnyawali, B.-U. Moon, J. Kieda, R. Karshafian, M. C. Kolios and S. S. H. Tsai, Soft Matter, 2017, 13, 4011-4016.

J. M. Zhang, E. Q. Li and S. T. Thoroddsen, J. Micromechanics Microengineering, 2014, 24, 35008.

R. Pimentel-Domínguez, J. Hernández-Cordero and R. Zenit, Opt. Express, 2012, 20, 8732.

H. Lee, H. Kim, H. Han, M. Lee, S. Lee, H. Yoo, J. H. Chang and H. Kim, Biomed. Eng. Lett., 2017, 7, 59-69.

J. Wu and R.-K. Li, Genes Dis., 2017, 4, 64-74. 
M. Seo, R. Williams and N. Matsuura, Lab Chip, 2015, 15, 3581-3590.

31

E. Y. Arashiro and N. R. Demarquette, Mater. Res., 1999, 2, 23-32.

Z. Nie, M. Seo, S. Xu, P. C. Lewis, M. Mok, E. Kumacheva, G. 53 M. Whitesides, P. Garstecki and H. A. Stone, Microfluid. Nanofluidics, 2008, 5, 585-594.

H. Lin, J. Chen and C. Chen, Med. Biol. Eng. Comput., 2016, 54, 1317-1330.

A. L. Klibanov, M. S. Hughes, F. S. Villanueva, R. J. Jankowski, W. R. Wagner, J. K. Wojdyla, J. H. Wible and G. H. Brandenburger, Magn. Reson. Mater. Physics, Biol. Med., 1999, 8, 177-184.

M. Parhizkar, M. Edirisinghe and E. Stride, RSC Adv., 2015, 5, 10751-10762.

A. Katiyar, K. Sarkar and P. Jain, J. Colloid Interface Sci., 2009, 336, 519-525.

K. N. Kumar and K. Sarkar, J. Acoust. Soc. Am., 2015, 138, 624-634.

Y. Ando, H. Tabata, M. Sanchez, A. Cagna, D. Koyama and M. P. Krafft, Langmuir, 2016, 32, 12461-12467.

T. Segers, L. De Rond, N. De Jong, M. Borden and M. Versluis, Langmuir, 2016, 32, 3937-3944. J. J. Kwan and M. A. Borden, Adv. Colloid Interface Sci., 2012, 183-184, 82-99.

A. A. Doinikov, A. Bouakaz and S. Member, 2011, 58, 981993.

S. V. Dalvi and J. R. Joshi, J. Colloid Interface Sci., 2015, 437, 259-269.

P. S. Epstein and M. S. Plesset, Appl. Sci. Res., 1950, 38, 133-141.

K. Sarkar, A. Katiyar and P. Jain, Ultrasound Med. Biol., 2009, 35, 1385-1396.

S. Shim, J. Wan, S. Hilgenfeldt, P. D. Panchal and H. A. Stone, Lab Chip, 2014, 14, 2428.

A. Volk, M. Rossi, C. J. Kähler, S. Hilgenfeldt and A. Marin, Lab Chip, 2015, 15, 4607-4613.

S. G. Charati and S. a. Stern, Macromolecules, 1998, 31, 5529-5535.

P. Thomas, S. Raghavan and S. Forry, Anal. Chem., 2011, 83, 8821-8824.

L. Xia, T. M. Porter and K. Sarkar, J. Acoust. Soc. Am., 2015, 138, 3994-4003.

220. 3716-3722.
R. Shih, T. Hall, C. Hill and A. P. Lee, 2012, 4, 12-20.

T. Segers and M. Versluis, Lab Chip, 2014, 14, 1705-14.

M. P. Kok, T. Segers and M. Versluis, Lab Chip, 2015, 15, 